\title{
Determination of the Dielectric Anisotropy of Cholesteric Liquid Crystals
}

\author{
JERZY HOFFMANN \\ Institute of Molecular Physics, Polish Academy of Sciences \\ Smoluchowskiego 17, 60-179 Poznań, Poland
}

(Received August 16, 2002)

\begin{abstract}
The method of determination of the main components of electric permittivity tensor for the cholesteric liquid crystals was proposed. The method was used to interpret the results obtained for pure 4-[(S,S)-2,3 epoxyhexyloxy]-phenyl-4-(decyloxy)-benzoate and the binary mixture of cyanobiphenyls.
\end{abstract}

PACS numbers: 61.30.Eb

\section{Introduction}

\subsection{Dielectric anisotropy of liquid crystals}

The electric permittivity is a tensor. The number of components of this tensor needed to describe the dielectric properties of a given material depends on its symmetry [1]. Among uniaxial liquid crystals, the simplest for description are materials in which the symmetry axis and the director $n$ are parallel. This kind of symmetry takes place in nematics, smectics A and B [2]. In this case, only two components of the electric permittivity tensor $\varepsilon_{i j}$ are needed to describe dielectric properties. These values can be determined by measuring $\varepsilon$ along the directions parallel $\left(\varepsilon_{\|}\right)$and perpendicular $\left(\varepsilon_{\perp}\right)$ to $n$.

\subsection{Symmetry and anisotropy of physical properties of cholesteric liquid crystals}

In cholesteric liquid crystals, which are also called chiral nematics, the direction of the vector $\boldsymbol{n}$ is a function of position [2]. The changes of this direction within the volume of liquid crystal are regular and draw a helix with a pitch, which depends on temperature. The cholesteric phase, similar to the nematic phase, has one symmetry axis. In this case, the helix axis $\boldsymbol{L}$, perpendicular to the director $n$, 
is the symmetry axis [2]. From this property follow two simplest measuring configurations in dielectric experiments:

- electric field $\boldsymbol{E}$ perpendicular to the helix axis $\boldsymbol{L}(\boldsymbol{E} \perp \boldsymbol{L})$ realized in the fingerprint texture,

- electric field $\boldsymbol{E}$ parallel to the helix axis $\boldsymbol{L}(\boldsymbol{E} \| \boldsymbol{L})$ realized in the grandjean texture.

This paper is devoted to description of the dielectric properties of the cholesteric phase in both mentioned systems of coordinates - one connected with the cholesteric helix, and another with the director $\boldsymbol{n}$.

\section{Experimental}

The results reported were obtained for the cholesteric and isotropic phases of two materials:

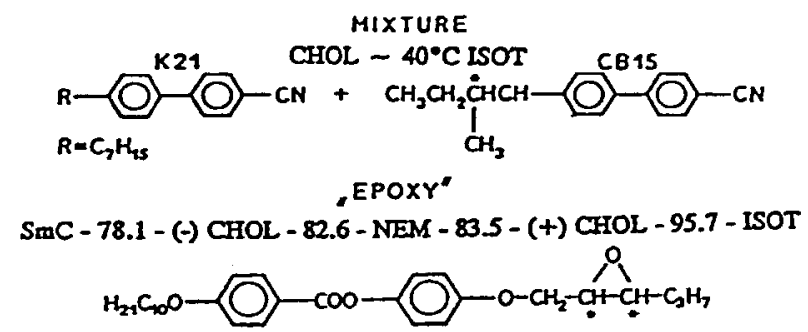

Electric permittivity was measured in cells of the sandwich type. The measurements were performed in two configurations: fingerprint and grandjean. The Mettler hot stage and the Unipan $650 \mathrm{H}$ Temperature Controller were used to stabilize the temperatures of samples with an accuracy of $\pm 0.01 \mathrm{~K}$.

A magnetic field about $1 \mathrm{~T}$ strength was used to unwind cholesteric helix. Electric capacity of measuring cell, which had been previously calibrated with liquids of a known electric permittivity, was used to calculate $\varepsilon$ of liquid crystal.

\section{Theoretical}

To determine the main components of the $\varepsilon_{i j}$ tensor in the case of uniaxial symmetry using the experimental data obtained in the two mentioned optional geometric configurations, one has to transform the $\varepsilon_{i j}$ tensor from the coordinate frame related to the main axes of molecule into the frame directly related to the experiment (laboratory frame) [3]. Let us denote the main values of the local electric permittivity ellipsoid as $\varepsilon_{1}, \varepsilon_{2}$, and $\varepsilon_{3}$, where $\varepsilon_{1}=\varepsilon_{2}=\varepsilon_{\perp}$ denotes the electric permittivity in the direction perpendicular to the long axis of the molecule (perpendicular to the local director $\boldsymbol{n}$ ), whereas $\varepsilon_{3}=\varepsilon_{\|}$expresses the electric permittivity in the direction of the long axis of molecule (parallel to the 
local director $\boldsymbol{n}$ ). After two successive transformations of the Cartesian coordinates assigned to the local ellipsoid of electric permittivity

$$
\left(x_{1}, x_{2}, x_{3} \rightarrow x_{1}^{\prime}, x_{2}^{\prime}, x_{3}^{\prime} \rightarrow x_{1}^{\prime \prime}, x_{2}^{\prime \prime}, x_{3}^{\prime \prime}\right),
$$

which consists in the rotation by the angle $\theta$ around the $x_{2}$ axis, and then the rotation by angle $\varphi$ around $x_{3}$ axis, one obtains the representation of the local $\varepsilon_{i j}$ tensor in coordinates in which symmetry axis is tilted to the direction of the director $n$ (angle $\theta$ ), and direction of $n$ can rotate around it ( $\varphi$ azimuthal angle). In the ideal, undisturbed structure of the cholesteric phase the angle $\theta$ describing tilt symmetry axis from the $n$ direction is equal to $90^{\circ}\left(\theta=90^{\circ}\right)$. The angle $\varphi$ and $x_{3}^{\prime \prime}$ are related to each other by the following formula:

$$
\varphi=\frac{2 \pi}{p} x_{3}^{\prime \prime}
$$

In practice, if the sample dimension along the direction of the $x_{3}^{\prime \prime}$ axis is much larger than the helical pitch $p$, the experiment gives an averaged value of $\varepsilon_{i j}$ along a particular direction. To calculate the mean values $\varepsilon_{i j}$ of the components of the local electric permittivity tensor one has to make averaging over the angle $\varphi$ for length corresponding to the helix pitch. Then the non-zero components of the $\varepsilon_{i j}$ have the following form:

$$
\overline{\varepsilon_{11}}=\overline{\varepsilon_{22}}=\frac{1}{2}\left(\varepsilon^{\prime}+\varepsilon_{2}\right), \quad \overline{\varepsilon_{33}}=\left(\varepsilon_{1}+\varepsilon_{3}-\varepsilon^{\prime}\right),
$$

where $\varepsilon^{\prime}=\varepsilon_{1} \cos ^{2} \theta+\varepsilon_{3} \sin ^{2} \theta$. For $\theta=90^{\circ}$ we obtain $\varepsilon_{i j}$ tensor in the final form

$$
\overline{\varepsilon_{11}}=\overline{\varepsilon_{22}}=\frac{1}{2}\left(\varepsilon_{3}+\varepsilon_{2}\right)=\frac{1}{2}\left(\varepsilon_{\|}+\varepsilon_{\perp}\right) \quad \overline{\varepsilon_{33}}=\varepsilon_{1}=\varepsilon_{\perp} .
$$

Tensor $\varepsilon_{i j}$ is diagonal in the laboratory frame $\boldsymbol{x}_{1}^{\prime \prime}, \boldsymbol{x}_{2}^{\prime \prime}, \boldsymbol{x}_{3}^{\prime \prime}$. It describes a uniaxial crystal with a symmetry axis parallel to $x_{3}^{\prime \prime}$, i.e. to the helix axis $\boldsymbol{L}$. After removing the helix structure (e.g. with a magnetic field applied along $\boldsymbol{x}_{2}^{\prime \prime}$ axis) the azimuthal angle is equal to zero for whole sample $\left(\varphi=0^{\circ}\right.$ and $\left.\theta=90^{\circ}\right)$. It gives the following components of the dielectric tensor:

$$
\varepsilon_{11}^{\prime}=\varepsilon_{3}=\varepsilon_{\|}, \quad \varepsilon_{22}^{\prime}=\varepsilon_{2}=\varepsilon_{\perp}, \quad \varepsilon_{33}^{\prime}=\varepsilon_{1}=\varepsilon_{\perp} .
$$

Now the $\varepsilon_{i j}$ tensor is diagonal in the laboratory frame $x_{1}^{\prime \prime}, x_{2}^{\prime \prime}, x_{3}^{\prime \prime}$ and describes a uniaxial crystal with a symmetry axis parallel to $\boldsymbol{x}_{1}^{\prime \prime}$, i.e. to the $n$ direction. This is the symmetry of the nematic phase.

\section{Results of calculations}

Applying the above considerations and Eq. (3) to the results of electric permittivity measurements of the cholesteric liquid crystal with grandjean configuration one can write

$$
\varepsilon_{\|}^{\mathrm{h}}=\overline{\varepsilon_{33}}=\varepsilon_{1}=\varepsilon_{\perp},
$$

where $\varepsilon_{\|}^{\mathrm{h}}$ denotes the permittivity along the helix axis $\boldsymbol{L}\left(x_{33}^{\prime \prime}\right)$. 
The measurements performed in the cholesteric samples of "fingerprint" orientation allow one to determine the component of $\varepsilon$ along a direction perpendicular to the helix axis $\varepsilon_{\perp}^{\mathrm{h}}$. Taking into account Eq. (3) one can write

$$
\varepsilon_{\perp}^{\mathrm{h}}=\overline{\varepsilon_{11}}=\overline{\varepsilon_{22}}=\frac{1}{2}\left(\varepsilon_{3}+\varepsilon_{2}\right)=\frac{1}{2}\left(\varepsilon_{\|}+\varepsilon_{\perp}\right) .
$$

Applying a strong magnetic field along a direction perpendicular to the helix axis $(\boldsymbol{B} \perp \boldsymbol{L})$ may cause unwinding of the cholesteric helix. After unwinding the helix, the electric permittivity changes from $\varepsilon_{\perp}^{\mathrm{h}}=(1 / 2)\left(\varepsilon_{\|}+\varepsilon_{\perp}\right)$ to $\varepsilon_{\|}$according to Eqs. (4) and (6). The magnetic field of the same orientation ( $\boldsymbol{B} \| \boldsymbol{L}$ ) does not cause any change in the value of $\varepsilon_{\|}^{\mathrm{h}}$.

To characterize the anisotropy of a given physical quantity one frequently introduces a parameter called "anisotropy", which is defined as a difference between the values of this quantity measured along the direction of symmetry axis, and perpendicularly to it [1]. Thus, the anisotropy of electric permittivity of cholesteric can be expressed as

$$
\Delta \varepsilon^{\mathrm{h}}=\varepsilon_{\|}^{\mathrm{h}}-\varepsilon_{\perp}^{\mathrm{h}} \text {. }
$$

Taking into account Eqs. (6) and (7) one can write

$$
\Delta \varepsilon^{\mathrm{h}}=\varepsilon_{\|}^{\mathrm{h}}-\varepsilon_{\perp}^{\mathrm{h}}=\varepsilon_{\perp}-\frac{1}{2}\left(\varepsilon_{\|}+\varepsilon_{\perp}\right)=\frac{1}{2}\left(\varepsilon_{\perp}-\varepsilon_{\|}\right) .
$$

Applying a magnetic field, which unwind the helix structure, leads to a transition from the cholesteric structure to the nematic structure. It means that the liquid crystal layer still maintains uniaxial symmetry, but the position of symmetry axis is changed by $90^{\circ}$. The symmetry axis of the nematic phase points to the direction of $n$ (which is parallel to the $\boldsymbol{X}_{1}$ axis). This means that the configuration used to determine electric permittivity along the direction perpendicular to the helix symmetry axis $\varepsilon_{\perp}^{\mathrm{h}}$, after unwinding, allows one to determine $\varepsilon_{\|}$of the nematic phase. Thus,

$$
\begin{aligned}
& \varepsilon_{\perp}^{\text {unw }}=\varepsilon_{3}=\varepsilon_{\|}, \\
& \varepsilon_{\|}^{\text {unw }}=\varepsilon_{1}=\varepsilon_{2}=\varepsilon_{\perp} .
\end{aligned}
$$

Defining "dielectric anisotropy" of the nematic phase as

$$
\Delta \varepsilon=\varepsilon_{\|}-\varepsilon_{\perp}
$$

and comparing (8) and (11) we obtain

$$
\Delta \varepsilon^{\mathrm{h}}=\frac{1}{2}\left(\varepsilon_{\perp}-\varepsilon_{\|}\right)=-\frac{1}{2}\left(\varepsilon_{\|}-\varepsilon_{\perp}\right)=-\frac{1}{2} \Delta \varepsilon, \quad \Delta \varepsilon^{\mathrm{h}}=-\frac{1}{2} \Delta \varepsilon .
$$

For liquid crystals composed of the same molecules the dielectric anisotropy in the cholesteric phase amounts to half of its value in the nematic phase and has the opposite sign (Fig. 1b, Fig. 2b).

\section{Results and discussion}

Figure 1 presents the results of dielectric measurements of the 4-[(S,S)-2,3 epoxyhexyloxy]-phenyl-4-(decyloxy)-benzoate (EPOXY) material as a function of 

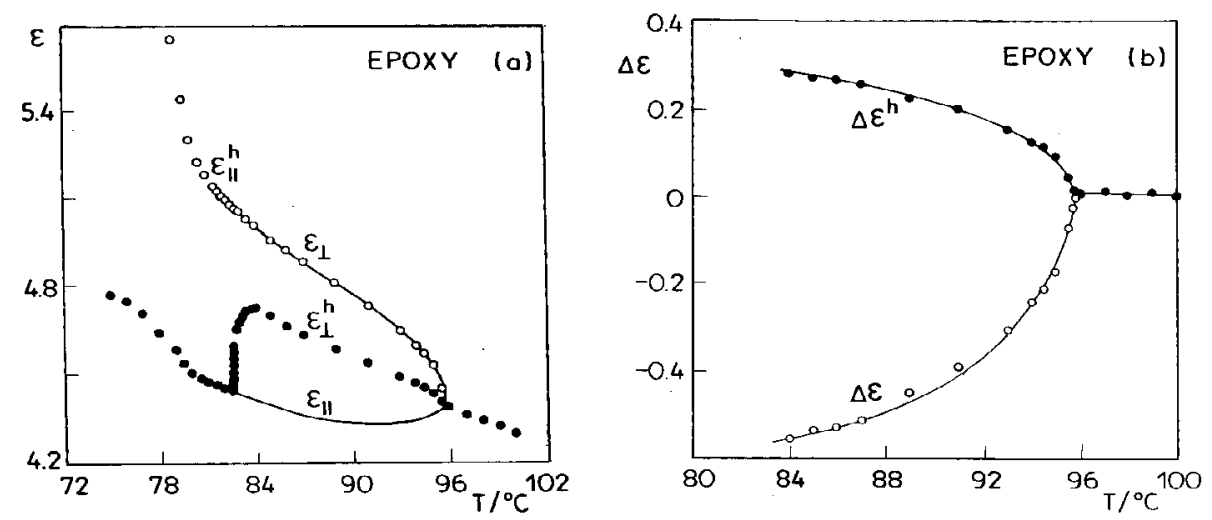

Fig. 1. Results of dielectric measurements of the EPOXY material (points), and results of calculations (lines). (a) $\varepsilon_{i j}$ permittivity tensor components, grandjean texture (open circles), fingerprint texture (filled circles). (b) Dielectric anisotropy $\Delta \varepsilon^{\mathrm{h}}$ and $\Delta \varepsilon$, results of calculations (open circles), results of experiment (filled circles).
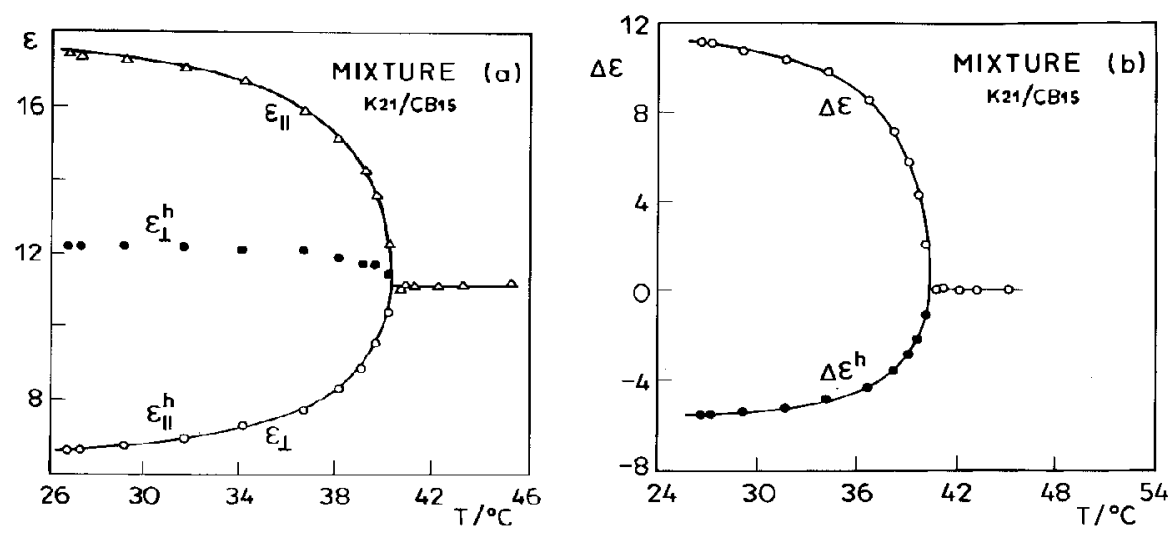

Fig. 2. Results of dielectric measurements of the MIXTURE material (mixture of cyanobiphenyls K21 and CB15). (a) $\varepsilon_{i j}$ permittivity tensor components, grandjean texture (open circles), fingerprint texture (filled circles), grandjean texture (open triangle), after unwinding helix structure (magnetic field applied). (b) Dielectric anisotropy $\Delta \varepsilon^{\mathrm{h}}$ and $\Delta \varepsilon$, results of experiment (open circles) with magnetic field applied, results of experiment (filled circles) without magnetic field.

temperature. This material reveals a quite uncommon feature reported by Dierking et al. [4], i.e. the temperature induced inversion of the helix pitch. According to Dierking a decrease in temperature from the isotropic phase produces the following molecular arrangement:

isotropic liquid $\stackrel{95.7^{\circ} \mathrm{C}}{\longrightarrow}$ dextrorotatory cholesteric $\stackrel{82.5^{\circ} \mathrm{C}}{\longrightarrow}$ (inversion of the helix pitch) nematic phase $\stackrel{81.6^{\circ} \mathrm{C}}{\longrightarrow}$ laevorotatory cholesteric $\stackrel{80.0^{\circ} \mathrm{C}}{\longrightarrow} \mathrm{SmC}^{*}$. 
Optical observations carried out during the dielectric measurements confirmed this sequence of phases with one exception only. During measurements of the $(\boldsymbol{E} \perp \boldsymbol{L})$ component, performed in $5 \mu \mathrm{m}$ cell giving the homeotropic orientation, a laevorotatory cholesteric phase was not observed at all, the nematic phase covered the range from $82.5^{\circ} \mathrm{C}$ to $80^{\circ} \mathrm{C}$. Figure 1a shows the results of calculations based on the model described above - the points corresponding to the nematic phase and the line calculated for the cholesteric phase are placed on the same curve.

The experimental results (points) and the results of calculations (solid lines) performed for the mixture of cyanobiphenyls (K21/CB15) is shown in Fig. 2a.

According to our expectations the calculated results agree well with the experimental data obtained in the presence of magnetic field (Fig. 2a).

It is worth noticing that $\Delta \varepsilon^{\mathrm{h}}$ may take positive or negative values depending on the structure of molecules forming the liquid crystal. For the cholesteric phase composed of biphenyl molecules (K21/CB15) (having the dipole moment parallel to the molecular long axis) $\Delta \varepsilon^{\mathrm{h}}$ is negative, whereas for the cholesteric phase of EPOXY material (having the dipole moment perpendicular to the molecular long axis) $\Delta \varepsilon^{\mathrm{h}}$ is positive (Fig. 1b, Fig. 2b).

\section{Conclusions}

The method for determination of the electric permittivity tensor components from the data obtained in cholesteric phase is proposed. The used model properly describes dielectric properties of liquid crystal in both cholesteric and nematic phases. The method enables one to determine the nematic like components of the $\varepsilon_{i j}$ (in the system of $n$ director coordinates) without unwinding the cholesteric helix. The method may be also used to predict the change in dielectric properties caused by inducing the cholesteric helix in nematics. These two features and the simplicity make the method useful in laboratories, which deal with the properties of liquid crystals in electric field.

\section{Acknowledgment}

This work was supported by the State Committee for Scientific Research in the framework of project 2P03B 12722 .

\section{References}

[1] J.F. Nye, Physical Properties of Crystals their Representation by Tensors and the Matrices, Clarendon Press, Oxford 1957.

[2] P.G. de Gennes, Physics of Liquid Crystals, Clarendon Press, Oxford 1974.

[3] J. Hoffmann, W. Kuczyński, J. Małecki, J. Pavel, Ferroelectrics 76, 61 (1987).

[4] I. Dierking, F. Giesselmann, P. Zugenmaier, W. Kuczyński, S.T. Lagerwall, B. Stebler, Liquid Crystals 13, 45 (1993). 